\title{
Ação antiarrítmica do isofluorano em cães submetidos à arritmias ventriculares induzidas por cloreto de bário
}

\author{
[Antiarrhytmic action of isoflurane in dogs submitted to ventricular arrhytmia by barium chloride] \\ W.L. Ferreira ${ }^{1}$, E.G. Aylon ${ }^{2}$, A.B. Carregaro ${ }^{3}$ \\ ${ }^{1}$ Curso de Veterinária - FOA - UNESP \\ Rua Clóvis Pestana, 793 \\ 16050-680 - Araçatuba, SP \\ ${ }^{2}$ Médica Veterinária autônoma \\ ${ }^{3}$ Departamento de Clínica de Pequenos Animais - UFSM - Santa Maria, RS
}

\begin{abstract}
RESUMO
Avaliou-se a ação antiarrítmica do isofluorano em cães submetidos a arritmias ventriculares pelo uso de cloreto de bário, utilizando-se de seis cães, machos e fêmeas, que receberam uma dose de $3 \mathrm{mg} / \mathrm{kg}$ de peso IV de cloreto de bário a 2,5\% (G1). O mesmo protocolo foi repetido, nos mesmos animais, sob anestesia geral com isofluorano (G2). Usou-se a eletrocardiografia computadorizada para avaliar o ritmo cardíaco, a duração e/ou amplitude das ondas e os intervalos eletrocardiográficos. Não se verificou alteração no ritmo cardíaco em G2, diferente de G1, que apresentou freqüentes arritmias ventriculares na forma de bigeminismo e taquicardia ventricular multifocal. Houve diferença significativa entre os grupos em relação à freqüência cardíaca nos minutos iniciais de observação, quando ocorreu aumento na freqüência cardíaca em G1. A utilização do isofluorano conferiu ação antiarrítmica em cães com arritmias induzidas pelo cloreto de bário, reforçando suas indicações a pacientes com risco considerável de desenvolvimento de arritmias ventriculares.
\end{abstract}

Palavras-chave: cão, arritmia ventricular, eletrocardiografia, isofluorano, cloreto de bário

\begin{abstract}
Antiarrhythmic action of isoflurane was evaluated in dogs submitted a ventricular arrhythmias by the use of barium chloride, using six dogs, males and females, that received $3 \mathrm{mg} / \mathrm{kgLW}$ intravenous dose of barium chloride $2.5 \%$ solution (G1). The same protocol was repeated on the same animals, after general anesthesia with isoflurane (G2). Computerized electrocardiography was used to evaluate the cardiac rhythm, waves duration and/or amplitude and electrocardiographic intervals. No alteration on the cardiac rhythm in G2 animals was observed, different from G1 animals, that showed frequent ventricular arrhythmias in bigeminism form, as well as mutifocal ventricular tachycardia. Differences between groups in relation of cardiac frequency in the observed initial minutes were showed, occurring an increase in cardiac frequency in $G 1$ animals. The utilization of isoflurane conferred antiarrhythmic action in dogs with arrhythmias barium chloride induced, reforcing its indication for patients with considerable risk of ventricular arrhythmias development.
\end{abstract}

Keywords: dog, ventricular arrhythmias, electrocardiography, isoflurane, barium chloride 


\section{INTRODUÇÃO}

Arritmias cardíacas são consideradas desordens na geração do impulso elétrico, na sua propagação ou ambos (Hoffman e Cranefield, 1964), muitas vezes observadas em pacientes com doença cardiovascular. Dentre as arritmias cardíacas, a taquicardia ventricular sustentada e a fibrilação ventricular são causas comuns de morte em cães (Muir et al., 1999). A taquicardia ventricular reduz o tempo disponível para o enchimento ventricular, diminuindo o débito cardíaco e aumentando a demanda de oxigênio do miocárdio (Jacobs, 1996). A utilização de modelos experimentais tem auxiliado no planejamento de estratégias diagnósticas e terapêuticas para as arritmias ventriculares (Germiniani e Germiniani, 1980; Schwartz, 1988; Dzielska-Olszak et al. 1998; Janse et al., 1998).

Em cães submetidos à anestesia geral, a ocorrência de arritmias cardíacas pode acontecer devido à administração de determinados fármacos. Nesse sentido, a anestesia inalatória tornou-se técnica comumente utilizada devido principalmente à segurança e a facilidade de controle do plano anestésico (Campagna et al., 2003).

O isofluorano é um éter metiletílico halogenado não-inflamável, de peso molecular 184.5 , ponto de ebulição em $48.5^{\circ} \mathrm{C}$ e pressão de vapor de aproximadamente $238 \mathrm{mmHg}$ a $20^{\circ} \mathrm{C}$, sendo utilizado como um anestésico volátil, cuja concentração alveolar mínima (CAM) em cães é de $1,28 \mathrm{~V} \%$ (Steffey e Howland, 1977). Apresenta coeficiente de solubilidade sangue/gás de 1.4 que, associado à sua alta potência, permite rápida indução anestésica (Massone, 2003). Segundo Morgan et al. (1996), os efeitos cardiovasculares do isofluorano em cães são mínimos, sendo o débito cardíaco preservado em concentrações de até 2 CAM e não sensibilizando o miocárdio à ação das catecolaminas.

O cloreto de bário é um sal solúvel, que determina intensa estimulação muscular, levando a despolarização em fibras musculares ventriculares, com ocorrência de extra-sístoles ventriculares (ESV) e fibrilação ventricular (Schuartsman, 1979). A administração do cloreto de bário em cães, gatos e coelhos tem sido usada para a indução experimental de arritmias ventriculares (Dawes, 1952; Dzielska-Olszak et al., 1998).

Este trabalho teve por objetivo avaliar os efeitos antiarrítmicos do isofluorano em cães submetidos à indução experimental de arritmias ventriculares por meio da administração intravenosa de cloreto de bário.

\section{MATERIAL E MÉTODOS}

Utilizaram-se seis cães, machos ou fêmeas, adultos, sem raça definida, considerados clinicamente sadios, os quais receberam ração comercial balanceada e água à vontade. Para a escolha dos animais, realizou-se triagem composta de exame físico, eletrocardiográfico e hematológicos (hemograma e bioquímica sérica). Os animais foram distribuídos em dois grupos (G). Em G1 fez-se indução das arritmias com o animal não medicado e em G2, indução das arritmias com o animal anestesiado. Os animais foram autocontroles, ou seja, os dois procedimentos foram realizados em todos os cães com intervalo de 15 dias entre as duas avaliações (autocontrole).

Em G1, visando a obtenção das arritmias cardíacas, canulou-se a veia cefálica para administrar lentamente, na forma de bolus, solução de cloreto de bário a $2,5 \%$ em água destilada, na dose de $3 \mathrm{mg} / \mathrm{kg}$. A dose foi definida de acordo com os resultados apresentados por Ferreira e Camacho (2001).

Para a execução do protocolo anestésico em G2 os animais foram mantidos em decúbito lateral esquerdo para canulação da veia cefálica. Procedeu-se a indução anestésica com administração intravenosa de $6 \mathrm{mg} / \mathrm{kg}$ de propofol ${ }^{2}$. O animal foi intubado e mantido sob anestesia geral inalatória pelo agente volátil isofluorano $^{3}$, por meio de equipamento específico em circuito circular valvular semifechado diluído em oxigênio ( $30 \mathrm{ml} / \mathrm{kg} / \mathrm{min})$. Ato contínuo, ajustou-se a concentração expirada do agente anestésico em $1,5 \mathrm{~V} \%$. Após o período de estabilização de 30 minutos, visando eliminar

\footnotetext{
${ }^{1}$ VETEC Química fina ltda, Duque de Caxias- RJ

${ }^{2}$ Propovan- Cristália Prod. Quím. Farm. Ltda. Itapira - SP

${ }^{3}$ Isoflorine- Cristália Prod. Quím. Farm. Ltda. Itapira - SP
} 
os efeitos do propofol, administrou-se a solução de cloreto de bário a 2,5\% e realizaram-se os registros eletrocardiográficos.

O estudo das arritmias foi conduzido por meio de registro eletrocardiográfico computadorizado ${ }^{4}$, de acordo com técnica descrita por Tilley (1992). Os registros foram realizados previamente e por 30 minutos após a administração de cloreto de bário. $\mathrm{Na}$ interpretação dos valores eletrocardiográficos foram utilizadas a derivação II e velocidade de $50 \mathrm{~mm} / \mathrm{seg}$., com calibração de amplitude um centímetro igual a um milivolt $(1 \mathrm{~cm}=1 \mathrm{mV})$.

Com relação ao ritmo cardíaco, foi criada uma escala (Tab. 1) para possibilitar a análise dos dados. Além do ritmo cardíaco, foram avaliados os seguintes parâmetros: duração (segundos) da onda P, intervalo PR, complexo QRS e intervalo QT; amplitude (milivolt) da altura da onda P e da onda $\mathrm{R}$; número de batimentos cardíacos por minuto.

Tabela 1. Esquema de classificação do ritmo cardíaco utilizado na análise das arritmias ventriculares obtidas com a administração intravenosa de solução de cloreto de bário a $2,5 \%$ em um grupo de seis cães

\begin{tabular}{l|c}
\hline Ritmo cardíaco & $\begin{array}{c}\text { Classifi- } \\
\text { cação }\end{array}$ \\
\hline Sinusal & 0 \\
ESV $<1$ por minuto & 1 \\
ESV $>1$ por minuto & 2 \\
Bigeminismo & 3 \\
ESV multiformes & 4 \\
ESV repetitivas duplas & 5 \\
ESV repetitivas duplas multiformes & 6 \\
ESV repetitivas triplos & 7 \\
ESV repetitivas triplos multiformes & 8 \\
\hline R em T & 9 \\
Fibrilação ventricular & 10 \\
\hline ESV:
\end{tabular}

ESV: extrassístoles ventriculares; $\mathrm{R}$ : onda eletrocardiográfica R; T: onda eletrocardiográfica T

Para avaliação do ritmo cardíaco fez-se uma análise bayesiana (Box e Tio, 1992), mostrando uma distribuição uniforme não informativa no intervalo das notas (entre 1 e 10) $\operatorname{com} \mathrm{P}(\theta)=$ 1/10 (MOOD et al. 1974). Para as demais variáveis usou-se o teste t para comparação de dados pareados (Zar, 1999).

${ }^{4}$ Módulo de aquisição de ECG- modelo ECG-PCTecnologia Eletrônica Brasileira- SP

\section{RESULTADOS}

Em relação às arritmias obtidas em G1, uma observação freqüente foi a presença de complexos ventriculares prematuros na forma de bigeminismo, caracterizado eletrocardiograficamente por uma ESV ocorrendo alternadamente com um batimento sinusal.

A taquicardia ventricular, que corresponde à presença de uma série contínua de ESV, ocorreu principalmente na forma multifocal, ou seja, com padrão irregular na configuração dos complexos QRS ectópicos.

Os resultados mostram em G2, com probabilidade maior que $99,9 \%$, que a administração intravenosa de solução de cloreto de bário a 2,5\% não teve resposta em cães anestesiados com isofluorano. Não houve variação nas notas conferidas aos ritmos no grupo anestesiado, permanecendo constante em todos os momentos em relação ao momento zero (ritmo sinusal). Essa ocorrência não possibilita uma análise estatística que compara os dois grupos (comparação entre uma constante e uma variável), mas confere uma diferença significativa entre os grupos.

Houve diferença significativa entre os grupos em relação à freqüência cardíaca nos quatro minutos iniciais (momentos 1 a 4), ocorrendo aumento na freqüência cardíaca em G1 (Tab. 2).

A ação do isofluorano resultou em aumento mais acentuado no período de repolarização ventricular quando comparado ao G1. Quanto à duração do intervalo QT, houve diferença significativa entre os grupos nos momentos 1 a 5 , com aumento em G2. Comportamento semelhante foi observado em relação à amplitude da onda $\mathrm{T}$.

Verificou-se ainda, que o cloreto de bário promoveu acentuação do peristaltismo intestinal em alguns animais nos dois grupos. 
Ação antiarrítmica do isofluorano em cães...

Tabela 2. Probabilidades do teste estatístico t para comparação pareada entre os grupos de cães $(n=6)$ não-medicados (G1) e anestesiado com isofluorano (G2)

\begin{tabular}{ccccccccc}
\hline Minutos & FC & Pseg & PmV & PRseg & QRSseg & RmV & QTseg & TmV \\
\hline 0 & 0,4039 & 0,5000 & 0,1816 & 0,1230 & 0,5000 & 0,1069 & 0,1816 & $0,0254^{*}$ \\
1 & $0,0080^{*}$ & 0,1816 & 0,3939 & 0,0802 & 0,0881 & 0,4396 & $0,0280^{*}$ & $0,0062^{*}$ \\
2 & $0,0241^{*}$ & 0,5000 & 0,4110 & $0,0237^{*}$ & 0,1074 & 0,1888 & $0,0004^{*}$ & $0,0036^{*}$ \\
3 & $0,0303^{*}$ & 0,0873 & 0,3178 & 0,3150 & 0,5000 & 0,1647 & $0,0017^{*}$ & $0,0037^{*}$ \\
4 & $0,0156^{*}$ & 0,1816 & 0,2377 & 0,1854 & 0,1816 & 0,3967 & $0,0077^{*}$ & $0,0057^{*}$ \\
5 & 0,0958 & 0,2004 & 0,2056 & 0,4423 & 0,1074 & 0,2993 & $0,0202^{*}$ & $0,0453^{*}$ \\
10 & 0,2206 & 0,2214 & 0,2462 & 0,2872 & 0,1816 & 0,1069 & 0,0682 & 0,1498 \\
15 & 0,1692 & 0,0790 & 0,2503 & 0,4314 & 0,1419 & 0,3907 & 0,0888 & 0,1086 \\
20 & 0,3684 & 0,0900 & $0,0436^{*}$ & 0,4087 & 0,1470 & $0,0499 *$ & 0,1212 & 0,1693 \\
30 & 0,4316 & 0,2680 & 0,3658 & 0,4024 & 0,5000 & 0,3187 & $0,0045^{*}$ & 0,0789 \\
\hline
\end{tabular}

FC: freqüência cardíaca; Pseg: duração em segundos da onda P; PmV: amplitude em milivolt da onda P; PRseg: duração em segundos do intervalo PR; QRSseg: duração em segundos do complexo QRS; RmV: amplitude em milivolt da onda R; QTseg: duração em segundos do intervalo QT; TmV: amplitude em milivolt da onda T.

* Diferenças significativas entre grupos $(\mathrm{P}<0,05)$.

\section{DISCUSSÃO}

A administração intravenosa de cloreto de bário a $2,5 \%$ na dose de $3 \mathrm{mg} / \mathrm{kg}$ mostrou-se eficiente no propósito de induzir arritmias ventriculares em cães não anestesiados. Os resultados confirmam os de Germiniani e Germiniani (1980) que relataram ser a administração de cloreto de bário excelente modelo experimental para avaliação da ação antiarrítmica ventricular de novos fármacos. O padrão multifocal das arritmias ventriculares conferiu mais importância ao modelo experimental, pois se aproxima de situações clínicas em que se necessita, muitas vezes, de imediata intervenção terapêutica.

A importância de se usar uma dose eficaz e segura de cloreto de bário fundamenta-se no trabalho de Mateos et al. (1997) que relataram as arritmias ventriculares como o mecanismo mais freqüente de morte súbita em cães. Trabalho anterior realizado por Sollmann (1977), concluiu que a administração de $8 \mathrm{mg} / \mathrm{kg}$ de cloreto de bário, por via intravenosa, causava arritmias difíceis de serem removidas com fármacos antiarrítmicos. Esses resultados reforçaram as evidências dos riscos proporcionados com a utilização do cloreto de bário em doses superiores à usada no presente estudo. Em nenhum momento foi reconhecida a ocorrência de arritmias supraventriculares ou alteração na velocidade de condução atrioventricular.
O período de 15 dias entre cada avaliação mostrou-se adequado como metodologia, tendo em vista que a meia vida do cloreto de bário em cães é inferior a 24 horas, conforme relatou Oehme (1979).

A não-indução de arritmias ventriculares nos cães anestesiados com isofluorano inviabiliza a utilização desse anestésico com a finalidade de constituir um modelo experimental para estudo de arritmias ventriculares induzidas com cloreto de bário em cães. A anestesia poderia ter $\mathrm{o}$ intuito de auxiliar na contenção dos animais e facilitar a condução do experimento e monitoração eletrocardiográfica durante a indução das arritmias.

O efeito antiarrítmico do isofluorano, neste caso, aparentemente está vinculado ao bloqueio da ação do cloreto de bário nas fibras miocárdicas. O cloreto de bário apresenta efeito direto na fibra miocárdica, proporcionando retardo na migração do potássio que, por sua vez, determina aumento na duração do potencial de ação, redução do potencial de repouso e ocorrência de despolarizações diastólicas em células ventriculares (Reid e Hecht, 1967). Não foi encontrada na literatura citação que descrevesse outra possível ação eletrofísiológica do isofluorano.

Vale ressaltar que a anestesiologia está sempre à procura de fármacos, associações e técnicas que melhor se adaptem aos mais diversos pacientes. 
Animais com risco considerável de desenvolvimento de arritmias ventriculares requerem anestesias que proporcionem estabilidade hemodinâmica, com fármacos isentos de potencial arritmogênico. Os resultados obtidos no presente estudo reforçam as indicações do isofluorano à pacientes que evidenciam tais riscos anestésicos.

O aumento significativo na freqüência cardíaca obtido em G1 pode ser atribuído à indução das arritmias, principalmente à taquicardia ventricular, nos momentos iniciais de observação. Esse fato também pode ser devido ao estresse de contenção, proporcionando aumento nos níveis de catecolaminas.

Neste estudo, verificou-se aumento no peristaltismo em alguns animais, ocorrência já observada por Schuartsman (1979). Da mesma forma, a diminuição da freqüência cardíaca nos momentos finais de observação pode ser em decorrência de um possível efeito colinérgico proporcionado pelo cloreto de bário, confirmando os estudos de Germiniani e Germiniani (1980) e justificando, também, a acentuação no peristaltismo.

\section{CONCLUSÃO}

A utilização do isofluorano conferiu ação antiarrítmica em face das arritmias induzidas com cloreto de bário em cães, reforçando suas indicações à pacientes com risco considerável de desenvolvimento de arritmias ventriculares.

\section{REFERÊNCIAS BIBLIOFRÁFICAS}

ANDRADE, S.F. Manual de terapêutica veterinária. 2.ed. São Paulo: Roca, 2002. 697p.

BOX, G.E.P.; TIO, G.C. Bayesian inference in statistical analysis. 3.ed. New York: Wiley Classical Library, 1992. p. 10-15.

DAWES, G.S. Experimental cardiac arrhythmias and quinidine-like drugs. J. Pharmacol. Exp. Ther., v.95, p.43-84, 1952.

DZIELSKA-OLSZAK, M.; POLAKOWSKI, P.; KUBIK-BOGUCKA, E. Evaluation of antiarrhythmic activity of captopril and enalaprilat in experimental cardiac arrythmias in rabbits. Pol. J. Pharmacol., v.50, p.225-231, 1998.

FERREIRA, W.L.; CAMACHO, A.A. Utilizacion del clorato de bario como modelo experimental de arritmias ventriculares en caninos monitorados por ecg computadorizada. Selec. Vet., v.9, p.246-252, 2001.

GERMINIANI, H.; GERMINIANI, C.L.B. Ação do cloridrato de amiodarona em arritmias ventriculares induzidas por cloreto de bário. Arq. Bras. Cardiol., v.34, p.503-508, 1980.

GORGELS, A.P.; VAN DEN DOOL, A.; HOFS, A. et al. Comparison of procainamide and lidocaine in terminating sustained monomorphic ventricular tachycardia. Am. J. Cardiol., v.78, p.82-83, 1996.

JACOBS, G.J. Cardiomyopathies: their classification and pathophysiologic features. Vet. Med., v.91, p.436-444, 1996.

JANSE, M.J.; OPTHOF, T.; KLEBER, A.G. Animal models of cardiac arrhythmias. Cardiovasc. Res., v.39, p.165-177, 1998.

MASSONE, F. Anesthesiologia veterinária: farmacologia e técnicas. 4.ed. Rio de Janeiro: Guanabara Koogan, 2003. 326p.

MATEOS, J.C.P.; KORMANN, D.S.; MATEOS, E.I.P. A terapêutica antiarrítmica previne a morte súbita? Rev. Soc. Cardiol. Est. São Paulo, v.7, p.240-254, 1997.

MOOD, M.A.; GRAYBILL, F.A.; BOES, D.C. Introduction to the theory of statistical. 3. ed. Tokyo: McGraw-Hill, 1974. p. 340-343.

MORGAN, G.E.; MIKHAIL, M.S. Inhalational anesthetics. In: Clinical Anesthesiology. 2.ed. Stamford: Appleton \& Lange, 1996. cap. 7, p. 109-127.

MUIR, W.W.; SAMS, R.A.; MOISE, N.S. Pharmacology and pharmacokinetics of antiarrhythmic drugs In: FOX, P.R.; SISSON, D.; MOISE, N.S. Textbook of canine and feline cardiology, 2.ed. Philadelphia: W.B. Saunders, 1999. p.307-330

OEHME, F. W. Toxicity of heavy metals in the environment. New York: Marcel Dekker, 1979. p.557-558. 
OLIVIER, N.B. Therapy Antiarrhythmic In: BONAGURA, J.D. Cardilogy, New York: Churchill Livingstone, 1987. p.219-249.

OMOIGUI, S. Manual de drogas usadas em anestesia. 2.ed. São Paulo: Santos, 1998. 566p.

REID, J.A.; HECHT, H.H. Barium induced automaticity in right ventricular muscle in the dog. Circul. Res., v.21, p.849-852, 1967.

SCHUARTSMAN, S. Metais, ametais e semimetais. In: _. Intoxicações agudas. 2.ed. São Paulo: Sarvier, 1979. p.256-257.

SCHWARTZ, P. Do animal models have clinical value ? Am. J. Cardiol., v.81, p.14D - 20D, 1988.

SMITH J.A.; GAYNOR, J.S.; BEDNARSKI, R.M. et al. Adverse effects of administration of propofol with various preanesthetic regimens in dogs. J. Am. Med. Assoc., v.202, p.1111-1115, 1993.
SOLLMANN, T. A manual of pharmacology and its applications to therapeutics and toxicology. 7.ed. Philadelphia: W.B. Saunders, 1977. p.478479.

STEFFEY, E.P.; ZINKL, J.; HOWLAND, D. Jr. Minimal changes in blood cell count and biochemical values associated with prolonged isoflurane anesthesia of horses. Am. J. Vet. Res., v.40, p.1646-1648, 1979.

TILLEY, L.P. Essentials of canine and feline eletrocardiography. 3.ed. Philadelphia: Lea e Febiger, 1992. 470p.

ZAR, J. Biostatistical analyisis. 4.ed. New Jersey: Prentice Hall, 1999. p.161-163. 